\title{
Analyzing and Modeling of Geo Spatial Effect on Radio Wave Propagation System Using Geospatial Technologies
}

\author{
Nigatu Bekele ${ }^{1}$, Biadgilgn Demissie ${ }^{2}$ \\ ${ }^{1}$ Department of Joint Military Staff College, Defense University, Mekelle, Ethiopia \\ ${ }^{2}$ Department of Geography and Environmental Studies, Mekelle University, Mekelle, Ethiopia \\ Email: Nigatubekele1983@gmail.com, biadgilgnd@gmail.com
}

How to cite this paper: Bekele, N. and Demissie, B. (2017) Analyzing and Modeling of Geo Spatial Effect on Radio Wave Propagation System Using Geospatial Technologies. Journal of Geographic Information System, 9, 777-795.

https://doi.org/10.4236/jgis.2017.96048

Received: October 19, 2017

Accepted: December 26, 2017

Published: December 29, 2017

Copyright $\odot 2017$ by authors and Scientific Research Publishing Inc. This work is licensed under the Creative Commons Attribution International License (CC BY 4.0).

http://creativecommons.org/licenses/by/4.0/

(c) (i) Open Access

\begin{abstract}
The research presented in this thesis reveals the level of rightness of the recurrence Prediction systems by correlated with geospatial effect. The Geospatial technology elements split up: Geographic Information System (GIS), Remote Sensing (RS) and Global Positioning System (GPS) consolidated into this technique in light of the fact that the vast majority of the components in radio wave propagation are geographic highlights. In this exploration, ICEPAC remote arranging programming is tried in a field test completed in Tigray and Afar district. The consequence show that, the Prediction programming doesn't put, day by day, regular and month to month topographical marvels into thought. Moreover, it doesn't demonstrate the correct area of the radio stations. Furthermore, the new proposed ICEPAC Calibration algorithm anticipates a good Signal quality for frequencies in the vicinity of $1.5 \mathrm{MHz}$ up to $30 \mathrm{MHz}$. The total result showed that Geographical Information Systems (GIS) are getting to be noticeably valuable apparatuses in accumulation, stockpiling, control and portrayal of Geo spatial information and also the RS and GIS situated Signal quality forecast can essentially enhance forecast quality contrasted with the hypothetical free space demonstration which does not consider any Geo spatial and neighborhood landscape highlights impacts.
\end{abstract}

\section{Keywords}

GIS, Remote Sensing, Signal, GPS, Geospatial Data

\section{Introduction}

The advancement of remote technology requires capable framework orchestrat- 
ing of cell Radio communication. The fundamental operations in the radio communication organize industry consolidate framework site conspicuous evidence and masterminding, signal quality estimations with scope estimation for the expansion of system. There are many models made by electrical experts in the earlier decades to separate Radio-com site sending and plan for cell frameworks [1]. However, out of these models none of them gives a keep going plan in light of the fact that each method has some particular repression in its application. The nonexclusive radio wave causing desire counts in perspective of PC databases or observational results gives simply estimated scope, which is not sensible for unequivocal framework layout.

The capability of radio wave inducing desire structure can be upgraded altogether, with viable limit of managing geo-spatial data through Remote Sensing and GIS frameworks [2]. Mechanized Mapping constitutes an important mostly toward supervising land resources, and mapped information is the consistent consequence of examination of remotely identified data [3]. The idea of the Radio organization relies upon the signal quality open at the customer's region. The signal begins from an arrangement of gathering devices sited at key zones over the scene. In any case, endeavoring to work out the best arrangement of radio wires over a broad region is troublesome and is dependent on different components, which consolidate arrive cover, scene undulations, building statures, creation and morphology. RS development and GIS can be melded into this technique in light of the fact that an expansive bit of the components in radio wave multiplication are geographic features.

This showing engages field planners to coordinate wave inciting ponders, and recognizes the perfect apex range, stature, and signal quality. In the present work, an undertaking has been made to consider in and around Tigray and Afar city of Ethiopia and its condition for the impact of soil resistivity, vegetation, water and land use and arrive cover on the radio authority tower site recognizing verification and orchestrating using RS, GIS and GPS. There are a combination of models made by electrical designers in the earlier decades to separate Radio and Radio-com Site Suitability in cell frameworks, yet none of them could be known as a last plan in light of the way that every single one of them has some particular repressions in its application [4].

Most repressions have relationship with the geographic features of the area to which they are being associated, for example, the blockages along radio wave spread ways, and the untidiness setback caused by different land covers, helping impacts on account of geological and higher soil resistivity and fitting site ID et cetera. Most repressions have relationship with the geographic features of the area to which they are being associated, for example, the blockages along radio wave spread ways, the untidiness setback caused by different land covers, helping impacts on account of geological and higher soil resistivity and fitting site ID et cetera. The idea of the organization relies upon the signal quality available at the customer's range. The signal starts from an arrangement of radio wires sited 
at crucial ranges over the scene. In any case, endeavoring to work out the best arrangement of gathering devices over a broad region is troublesome and is liable to different parts, which consolidate arrive cover, scene undulations, building statures, creation and morphology [4].

The essential operations in the communication arrange industry incorporate system site distinguishing proof and arranging, signal strength quality estimations with scope estimation for the extension of framework [5]. There are many models created by electrical specialists in the previous decades to break down communication site organization and plan for cell systems, for example, Lee model [6].

However, these models don't give a last arrangement on the grounds that every system has some specific impediment in its application. The non-specific radio wave proliferation forecast calculations in view of PC databases or observational outcomes give just surmised scope, which are not reasonable for definite system outline. The proficiency of radio wave spread Prediction framework can be enhanced extraordinarily, with intense ability of dealing with geo-spatial information through RS and GIS strategies.

Signal Strength quality anticipated by joining of ground study techniques and by utilizing model investigation that consider the effect of Geo spatial wonders, (for example, arrive utilize/arrive cover, atmosphere variety) are vital for the variety in Signal Strength quality. At the point when the Signal Strength strikes the surface of a building, it might be diffracted or consumed, this is an imperative thought in the scope arranging of a radio system. RS/GIS arranged Signal Strength quality Prediction display strategy may enhance the forecast quality contrasted with the hypothetical free space demonstrate which does not consider any Geo spatial impacts [6].

The multi otherworldly and stereo satellite information in conjunction with GIS/RS/GPS systems can be used to figure appropriate beneficiary site and procedures for a viable communication arranging and improvement. The present proposition work is essential to help communication framework fashioners in different climate determining and communicate stations, which confront the test of foreseeing and adjusting for this possibly genuine climate related impacts on communication.

\section{Study Area}

The study conducted in two places, one inside "Tigray" region, "Tigray" is located at the northern limit of the central highlands of Ethiopia (Adwa, Quiha, Wukro) and the other inside Afar area, Ethiopia. Tigray Region lies in Northern Ethiopia and has a rugged terrain, ranging from 400 to $4000 \mathrm{~m}$ above sea level. Tigray has international boundaries with Sudan and Eritrea to the west and north, and borders with the Afar and Amhara regions of Ethiopia to the east and south. The climate is predominantly semi-arid. In most parts of the region, the rainfall season lasts for only three months from mid-June to mid-September. The land- 
form is complex composed of highlands (in the range of 2300 - 3200 meters above sea level, (mask), lowland plains (with an altitude range of <500 - 1500 mask), mountain peaks (as high as 3935 masl) and high to moderate relief hills (1600 2200 masl). Tigray has diversified ago-ecological zones and task each with distinct soil, geology, vegetation cover and other natural resources.

The climate is generally Sub-tropical with an extended dry period of nine to ten months and a maximum effective rainy season of 50 to 60 days.

The Figure 1 for location map of the study area is listed below and shown in the appendices.

The Figure 1 shows the location map of the study area. The total number of study area are two locales, one inside Tigray Region (Adwa, Quiha, Wukro ) and the other inside Afar area, Ethiopia.

\section{System, Data, Materials and Software Utilized}

\section{Data}

In this exploration diverse sorts of information are utilized. These incorporate meteorological information, records of Signal Strength quality, satellite image and ground study information. The meteorological information was acquired

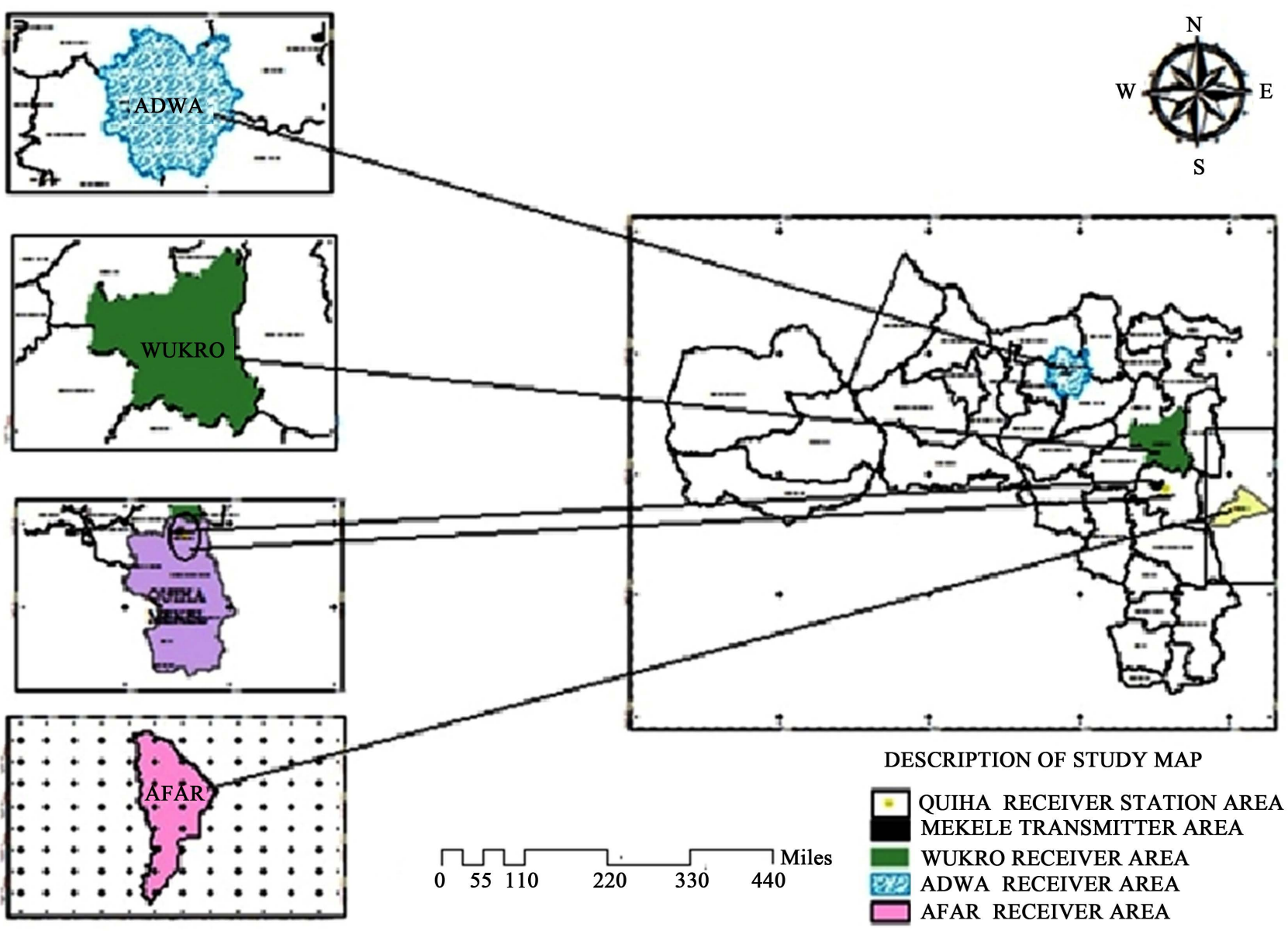

Figure 1. Location map of the study area. 
from the Tigray Meteorological Agency (Ten years Recorded Data) whereas the records of the Signal Strength quality (ten years recorded data) were gotten from the Ministry of National Defense, Northern Command Communication Center. The meteorological information is utilized for breaking down the impact of the HF engendering over the examination zone. The recorded Signal Strength quality information of the examination destinations are utilized for breaking down the Signal Strength quality for all beneficiary station over the investigation range.

The satellite images are removed from Google Earth. This is utilized for breaking down land utilize/cover. The ground study information was utilized for precision evaluation over the ground for exactness appraisal over the investigation range.

\section{Materials and Programming}

In this exploration distinctive materials and programming were utilized. The materials incorporate HF-Receiver, HF-Radio, GPS-Receiver and Signal-Meter, HF-Receiver reception apparatus. It was accustomed to accepting radio signal. The HF-Radio is utilized to for communication.

The GPS-Receiver was accustomed to gathering X and Y focuses. Signal-Meter is utilized break down Signal Strength and measured signal strength in the receiver station. The products that are utilized as a part of the investigation are ERDAS.

Imagine 9.1, ArcGIS 10.5, ICEPAC, SPSS, R-Software (Geo-Statically Analysis) and Radiation Software.

The ERDAS Imagine is programming which is created by Leica for image preparing. ArcGIS is a GIS programming bundle created by ESRI for different GIS applications, in this investigation it was utilized to Geospatial examination and Geo statically analysis. The ICEPAC is utilized for Signal Strength Prediction.

\section{Research Methodology}

In this examination, a technique in light of GIS/RS has been utilized. The proposed technique to assess the ICEPAC show includes a few stages. The accumulated remotely detected image information are proposed to be dissected utilizing GIS and RS Software, while alternate sorts of information were analyzed in connection to the outcomes got from remote detecting information investigation.

The Figure 2 for research methodology is listed below and shown in the appendices.

The Figure 2 shows the overall objective and technique of how to finalizing the stated research work.

\section{Results and Discussion}

\section{Radio Wave Propagation Models}

This piece of the part introduces the consequence of examination of the diverse Signal Strength Prediction models. There are assortments of models created in 


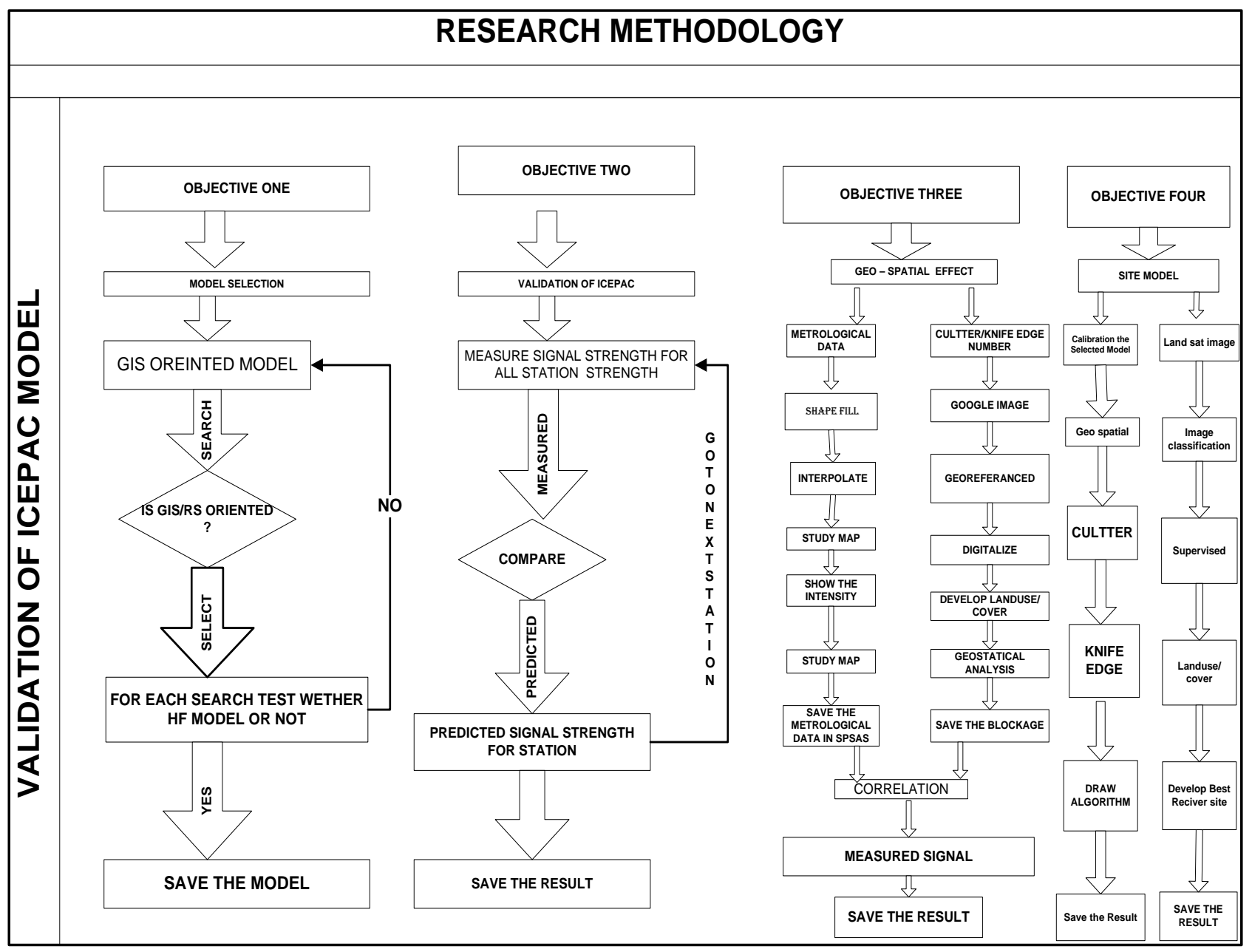

Figure 2. Research methodology.

the previous decades to examine wave engendering in cell systems. Yet, none of them could be known as a last arrangement in light of the fact that each one of them has some specific confinements in its application.

Most restrictions have associations with the geographic highlights of the area to which they are being connected, for instance, the blockages along radio wave engendering ways, the messiness misfortune caused by various land covers, and so forth. GIS can mastermind such area based data proficiently and precisely. So normally GIS is considered to help in taking care of issues in planning a cell organizes advantageously and proficiently [5].

With a specific end goal to plan and streamline cell radio systems, precise way misfortune models, are required which utilize fit for taking care of topographical data framework. When all is said in done there are two general classifications of proliferation models accessible which are to be specific observational models and physically-based models. Observational way misfortune models are generally utilized for the arranging and enhancement of cell systems. They treat the way misfortune related with a given full scale cell as subject to remove, gave that the earth encompassing the base station is level and genuinely uniform. 
In result, the scope zone anticipated by these models for a disengaged base station are approximated as roundabout; despite the fact that this is obviously off base, it is helpful for framework dimensioning purposes.

Experimental models were made by fitting proper numerical capacities to broad arrangements of measured way misfortune information; no endeavor on physical models of superseding proliferation components. Amendment terms and factors are frequently inferred for specific spread conditions, frequencies and radio wire statures, and utilize, arrive cover to such an extent that the mistake between the model and the estimations is limited.

In view of the demonstrating data acquired from analysts, books and going to various locales, we can partition the models into three fundamental classes regularly, Empirical, Deterministic models, stochastic model. In light of these diverse models are acquired HATA, COMSITE, OKUMURA, LEE, ICEPAC, and ASAPS. Out of these, the Empirical models are chosen for this exploration. Be that as it may, the geological division of working area of ASAPS in southern side of the equator and its recurrence limits is out of HF run. Be that as it may, the ICEPAC show that the radio transmission station is working, the model is working underneath northern half of the globe and its recurrence in the scope of (1.5 up to $30 \mathrm{MHz}$ ) which is in the HF extend. In light of this model, the forecast and its deliberate wave has been looked at.

\section{Comparisons of GIS Oriented Models (Result)}

Table 1 for GIS and Remote Sensing Propagation Model is listed below and shown in the appendices.

From Table 1 shows that, the comparison of GIS models, the result shows that HATA, COMSITE, OKUMURA, LEE, models were worked on Very High Frequency range whereas ICEPAC, and ASAPS were worked on the High Frequency range. However, Out of these, the Empirical models are chosen for this exploration. Despite that, the geological division of working area of ASAPS in southern side of the equator and its recurrence limits is out of HF run. Be that as it may, the ICEPAC show that the Ministry of National Defense is working,

Table 1. GIS and remote sensing propagation model.

\begin{tabular}{cccc}
\hline & & \multicolumn{2}{c}{ Classical model for VHF } \\
\hline Model type & Frequency range & Application & Developed by \\
\hline HATA & $>2500 \mathrm{MHz}$ & The urban land, shrub, open land & Hata (1980) \\
COMSITE & Up to $2000 \mathrm{MHz}$ & For vegetation cover & Li Qing \\
OKUMRA & $150-1920 \mathrm{MHz}$ & An irregular terrain and environmental clutter & Okumra et al. \\
LEE & $1-100 \mathrm{MHz}$ & Forest area & Y. S. Meng Y. H. Lee \\
MODEL TYPE & Frequency range & Current HF Propagation & Developed by \\
ICEPAC & $1.5-30 \mathrm{MHz}$ & Application & ITU \\
ASAPS & $\mathbf{1}-\mathbf{4 5 \mathrm { MHz }}$ & Through ionosphere & ITU
\end{tabular}


the model is working underneath northern half of the globe and its recurrence in the scope of ( 1.5 up to $30 \mathrm{MHz}$ ) which is in the HF extend. In light of this model, the forecast and its deliberate wave has been looked at.

\section{Discussion of the Resulting Model \\ ICEPAC Model:}

For a long time, various associations have been utilizing the HF range to impart over long separations. It was perceived in the late thirties that these communication frameworks were liable to varieties in execution. The viable operation of long-remove HF frameworks expanded in extent to the capacity to anticipate varieties in the ionosphere, since such capacity allowed the choice of ideal frequencies, receiving wires, and other circuit parameters. An overall system of particle sounds was set up to gauge ionosphere parameters. Overall clamor estimation records were taken and watched varieties in Signal Strength and amplitudes were recorded over different HF ways [7].

The consequences of this exploration built up that most varieties in HF framework execution were straightforwardly identified with changes in the ionosphere, which thus are influenced in an unpredictable way by sun based action, occasional and diurnal varieties, and scope and longitude. A treatise of ionosphere radio proliferation was distributed by the Central Radio Propagation Laboratory (CRPL) of the National Bureau of Standards [7].

This report delineated the cutting edge in HF spread. Manual systems were given for breaking down HF circuits of short, halfway, and long separations. As the manual techniques were relentless and tedious, different associations created PC projects to dissect HF circuit execution.

The short-distance model evaluates all possible ray paths for the circuit, including high and low angle modes; E, Fl, and F2 modes; above the MUF modes; and sporadic-E modes. Losses include regular D-E absorption, deviate losses, and sporadic-E losses. The CCIR-262 loss is basically for F2 modes. The noise at the receiver site is evaluated and combined with signal statistics to estimate the SNR statistics. An extension of the single-hop model to long paths would lead to the Prediction that failure of propagation at any of the reflection areas would cause propagation to fail altogether. Empirically, however, it has been found that propagation does not fail until the ionosphere either fails to launch a sky-wave or does not permit sky-wave reception; i.e., these are control areas about $2000 \mathrm{~km}$ from each end of the path [8].

\section{Evaluation of the Propagation Model (ICEPAC)}

The correlation is done in three ways; month to month, occasional and every day. Month to month Prediction demonstrates that diverse outcome for every recipient station. It demonstrates that Quiha recipient station result is low contrasted with others. Quiha recipient station represents $10 \%$, wukro 20\%, Adwa 30\%, finally Afar $40 \%$ of Signal Strength misfortunes. In the event of Occasional forecast it is seen that spring has great Signal Strength quality and summer has been observed to be the slightest in Signal Strength quality. 
Every day forecast it is seen that for $(1-3)$ hours Signal Strength misfortune is $26 \%$, for $(3-6)$ hours it is $24 \%$, for $(6-9)$ hours Signal Strength misfortune is $23 \%$ and for $(9-12)$ hours it is, $27 \%$. The outcomes demonstrates that amid evening, for (1 - 3) hours Signal Strength misfortune is $24 \%$, for (3 - 6) hours Signal Strength misfortune is $23 \%$, for (6 - 9) hours Signal Strength misfortune is $26 \%$ and (9-12) hours it is $27 \%$. Amid the day time from (6-9) Hours, there is a decent communication and amid evening time from $(3-6)$ hours there is a decent wave engendering. When all is said in done this demonstrates the Prediction display has showed signs of improvement motion than the deliberate signs. This implies the Prediction programming doesn't put, day by day, regular and month to month topographical marvels into thought. Furthermore, it doesn't demonstrate the correct area of the radio receiver stations.

In the meantime it additionally doesn't consider the ionospheric development in the climate. This implies amid around at dawn and around day down the movements of particles is unfaltering. Communication amid such time is feeble.

The arrangement is increment recurrence, amid evening time and diminishing recurrence amid day time. Wind, stickiness, daylight, rain fall, air temperature and substance of the environment can together either constrain (enormously lessen wave spread making typical communications to a great degree troublesome) or accelerate radio interchanges Precipitation in the climate has its most noteworthy impact on the recurrence ranges. The accompanying outcome demonstrates metrological impact on measured Signal Strength quality [9].

\section{Result: The Effect of Geospatial Phenomena}

The following effect of Geospatial phenomena analysis were carried out for better appreciation of the facts on ground, this helps with the understanding and in turn provides solutions to problems.

The Figures 3-10 for the various effects of Geospatial phenomena are listed below and shown in the appendices.

The Figures 3-10 shows the total number and types of clutter and Knife around the buffer zone of each receiver station which was affected signal strength at the receiver station.

\section{QUIHA Receiver Station}

Figure 3 shows land use of Quiha receiver station.

The Figure 3 below shows the total number and types of clutter and Knife around the buffer zone of QUIH receiver station which were affected signal strength at the receiver station. However, residential area encircled the receiver antenna.

Figure 4 shows effect of Knife-edge and Clutter number at Quiha receiver station.

The Figure 4 below shows the impact of clutter and cut edge around the radio antenna recipient stations, the central point shown that were the receiving antenna introduced, be that as it may, the distinctive point around the radio antenna collector stations appeared, the diverse clutter and cut edge components 


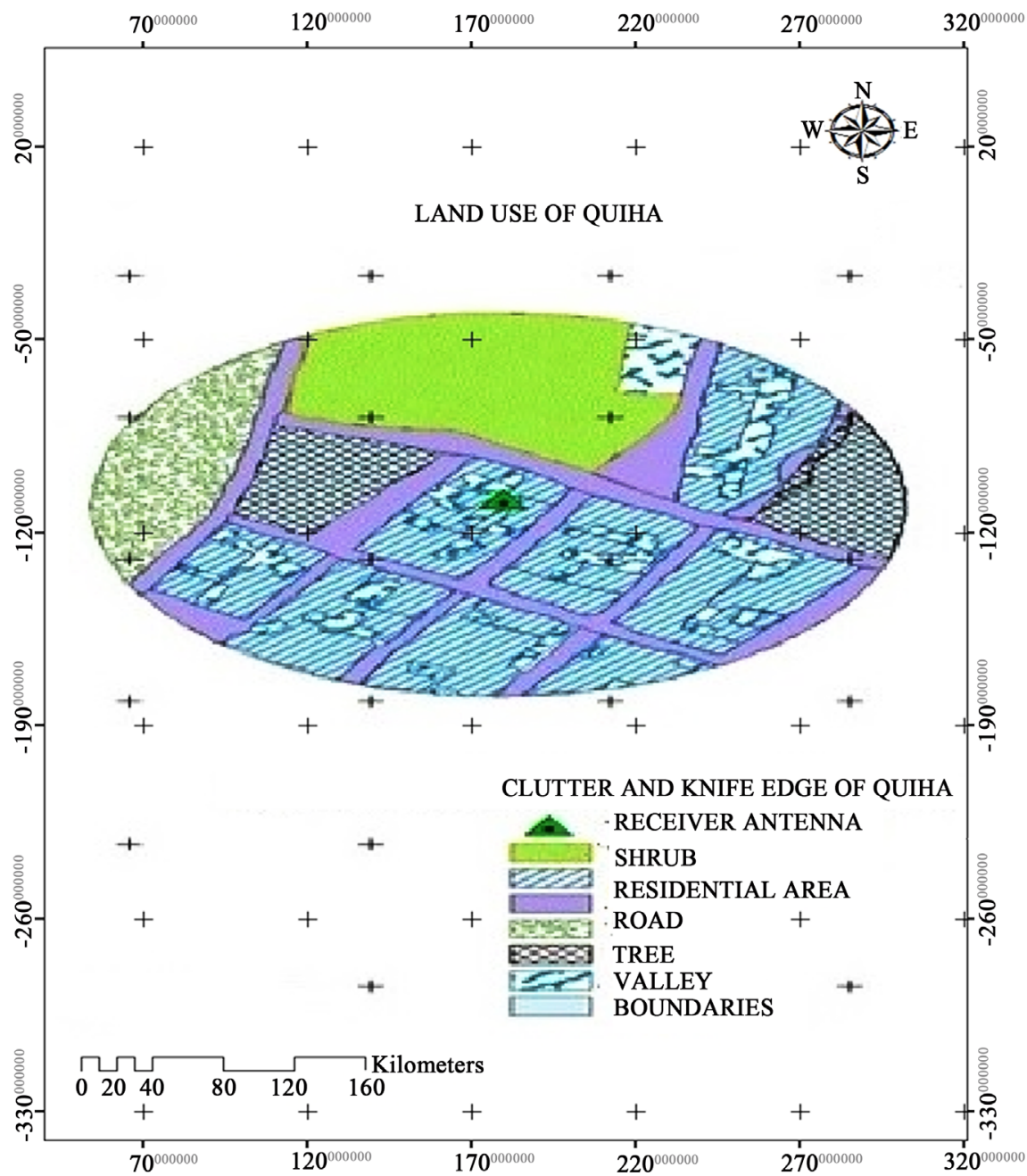

Figure 3. Land use of Quiha receiver station.

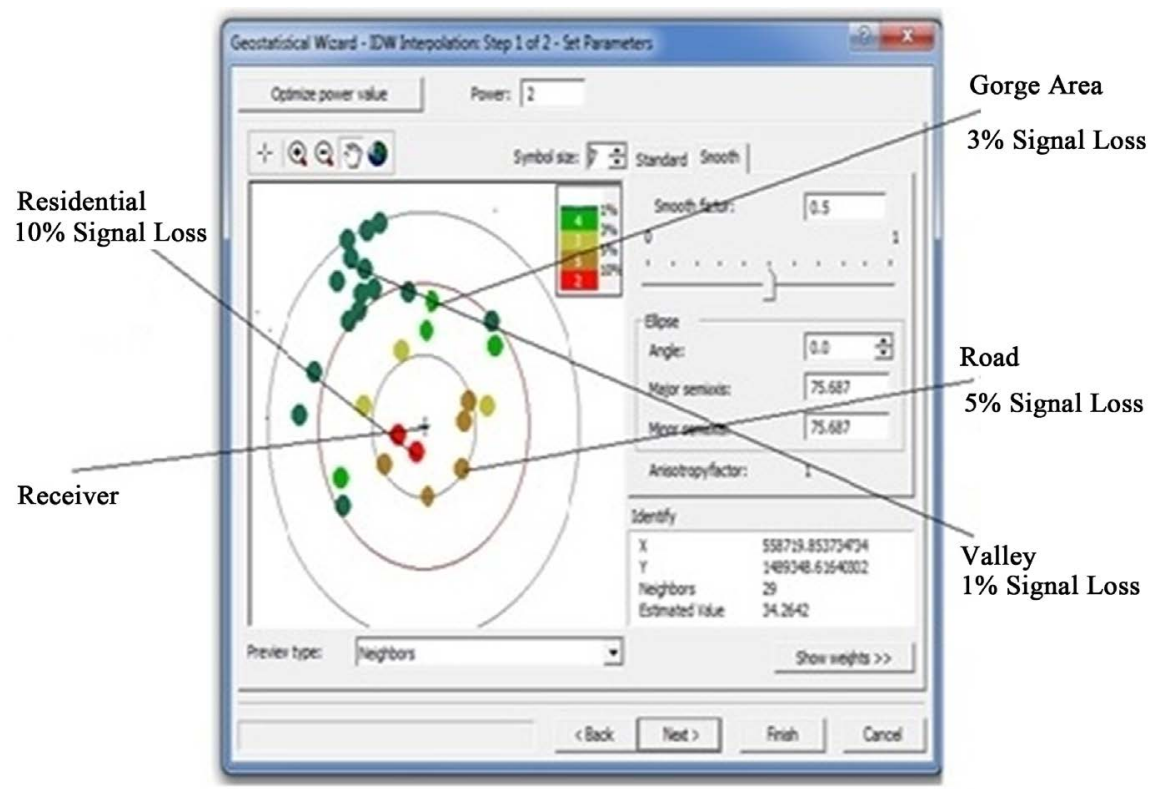

Figure 4. Effect of knife-edge and clutter number at Quiha receiver station. 


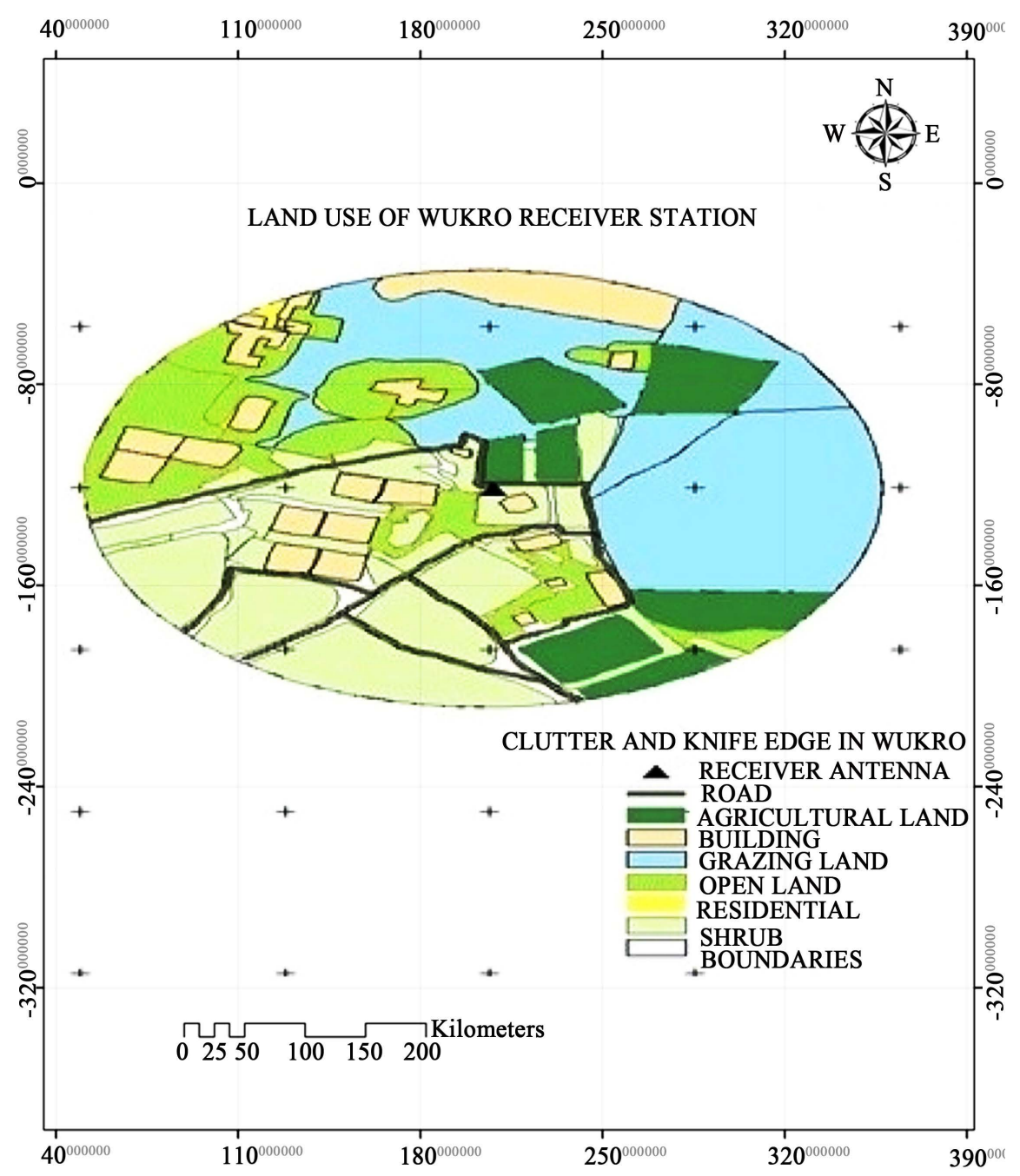

Figure 5. Land use of Wukro receiver station.

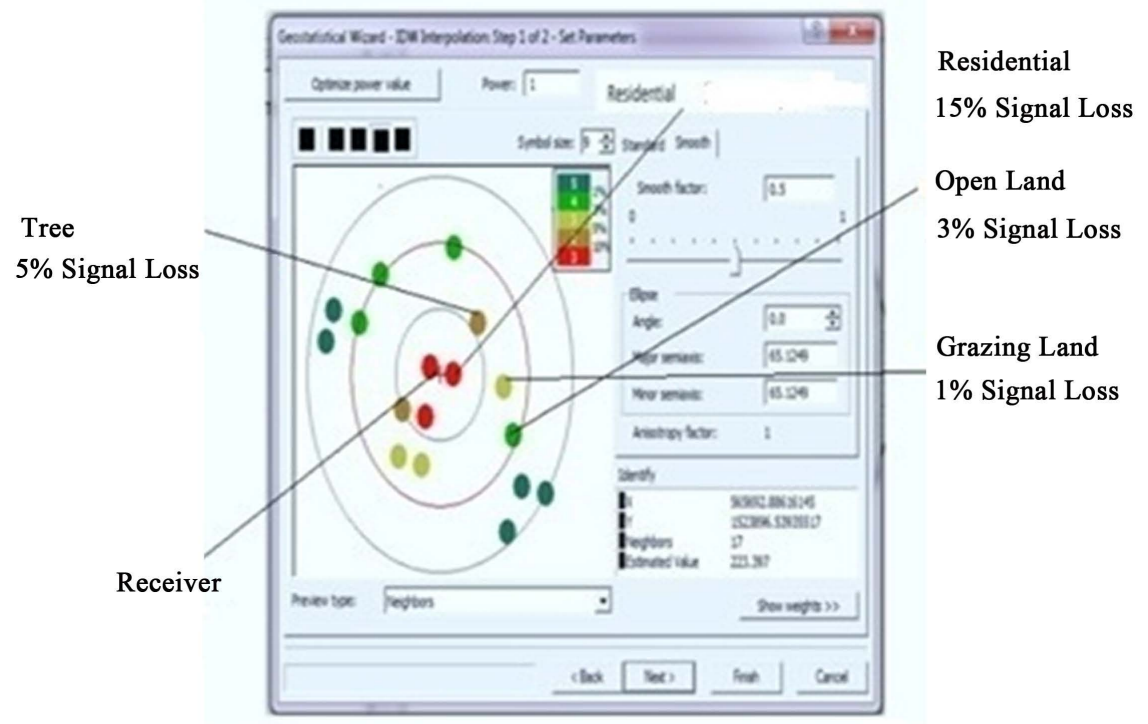

Figure 6. Effect of knife-edge and clutter number at Wukro receiver station. 


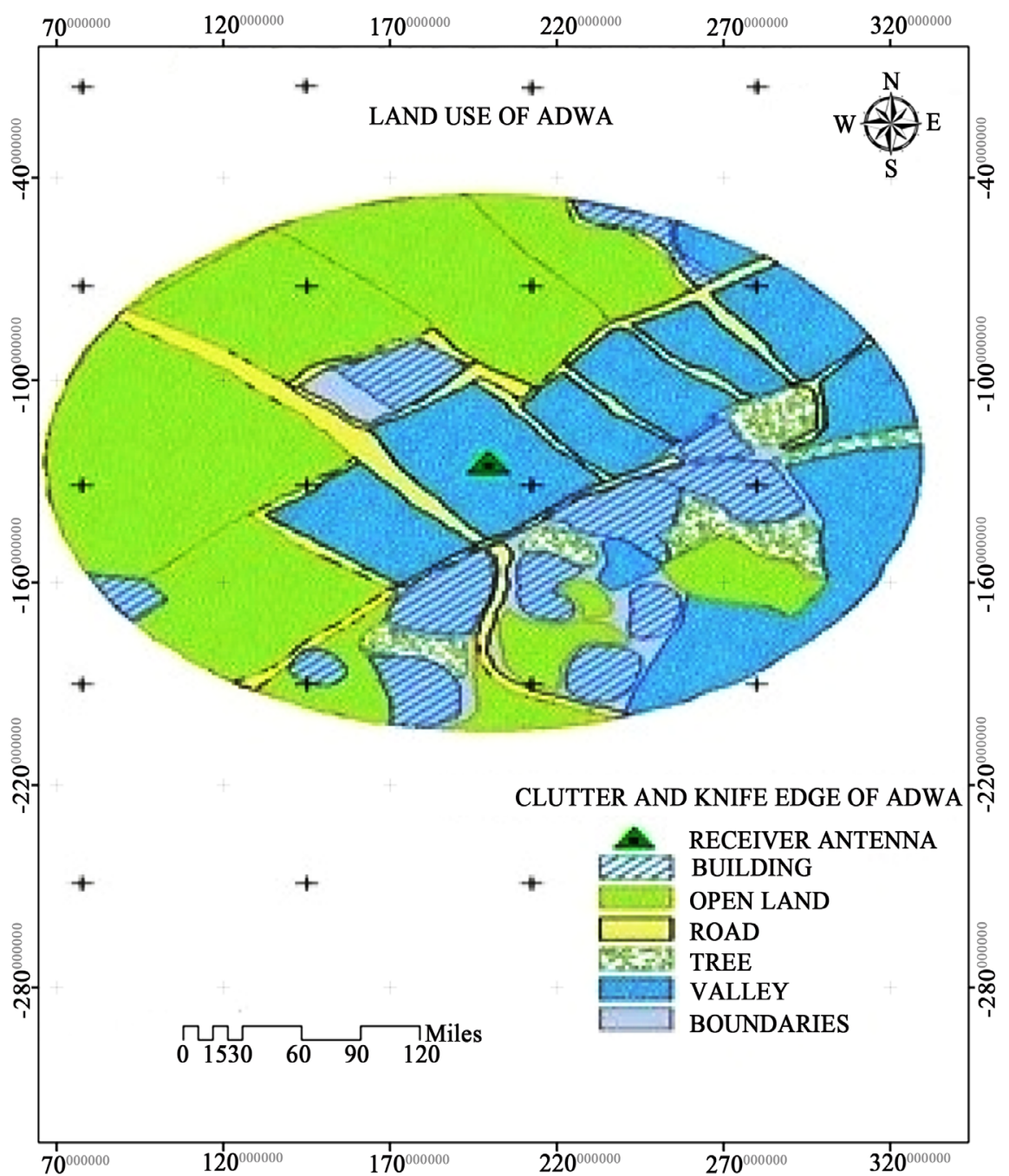

Figure 7. Land use of Adwa receiver station.

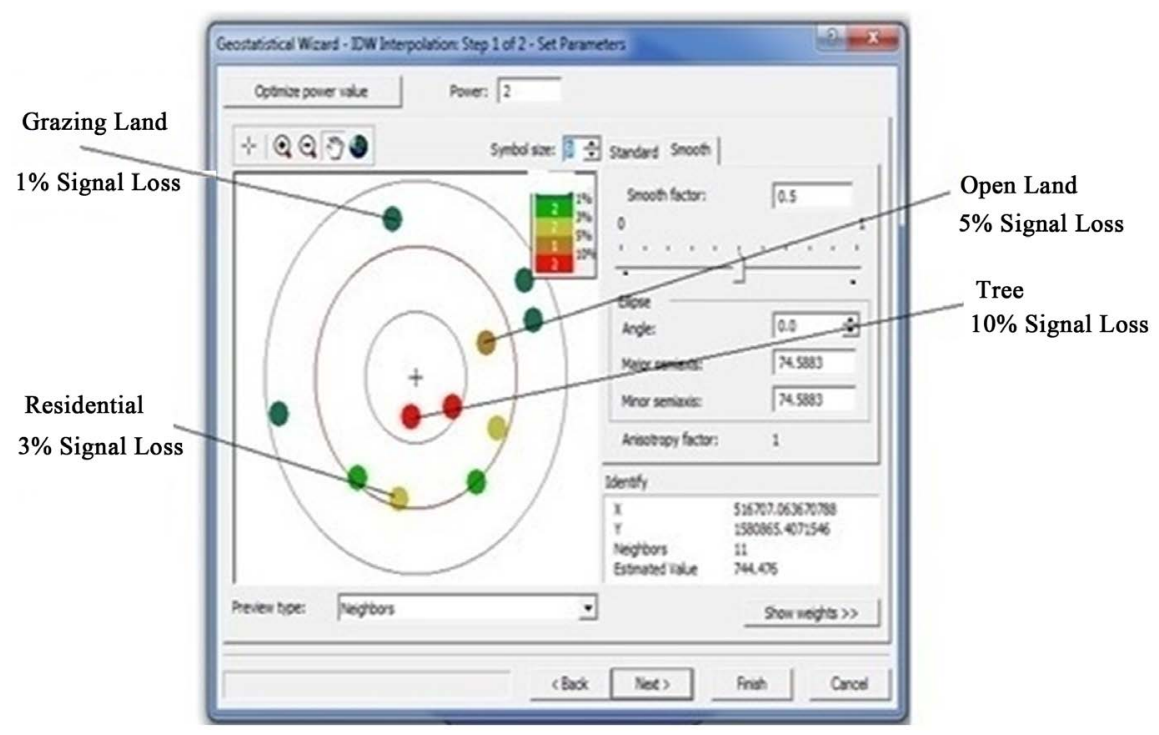

Figure 8. Effect of knife-edge and clutter number at Adwa receiver station. 


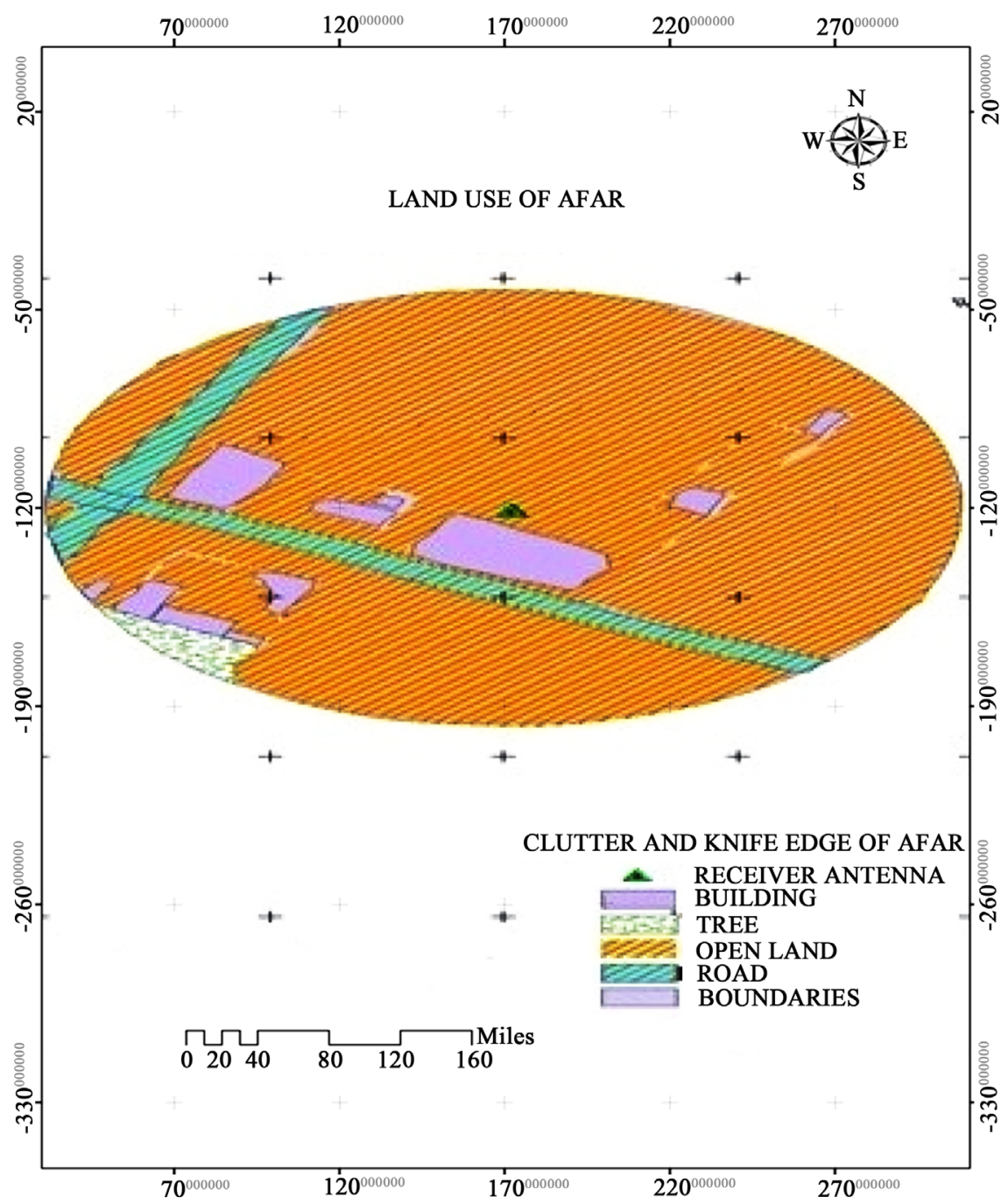

Figure 9. Land use of afar receiver station.

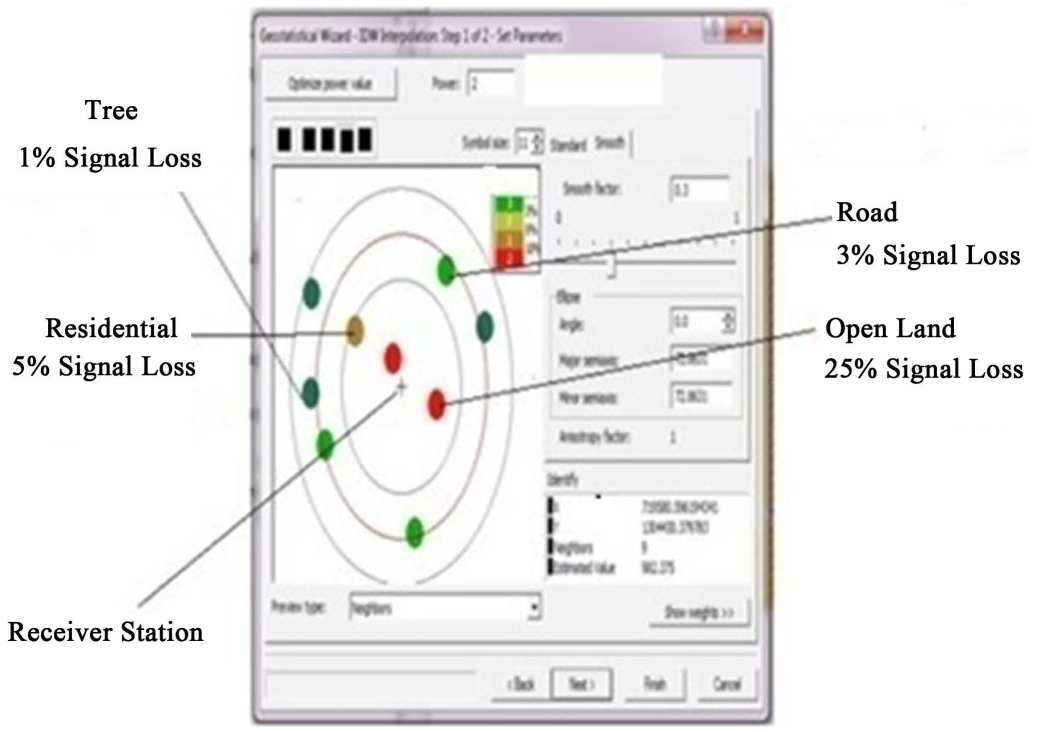

Figure 10. Effect of knife-edge and clutter number at afar receiver station. 
were influenced, the approaching signal strength quality in the radio antenna. The size of decreasing signal strength quality depends on the sorts of and the number of clutter and cut edge impact around the receiving antenna. From the graph, it is shown that the signal loss over the land use and land cover pattern of the Quiha receiver station has been recorded $10 \%$ loss with residential, $5 \%$ loss with road, $3 \%$ loss with gorge, $1 \%$ loss due to valley. However, the residential area is more influenced Signal Strength quality compared from others. Further more technically speaking Quiha receiver station mostly affected by atmospheric problem.

\section{WUKRO Receiver Station}

Figure 5 shows land use of Wukro Receiver Station.

The Figure 5 above shows the total number and types of clutter and Knife around the buffer zone of WUKRO receiver station which were affected signal strength at the receiver station. However, residential area encircled the receiver antenna.

Figure 6 shows effect of Knife-edge and Clutter number at Wukro receiver station.

The Figure 6 above shows the signal loss over the land use and land cover pattern of the Wukro receiver station has been recorded 15\% loss with residential, $5 \%$ loss with trees, $1 \%$ loss with grazing land and $3 \%$ loss due to open land. However, the figure showed that the receiver antenna mostly affected by residential area around the antenna buffer zone. In order to have good signal strength in the receiver station, the entire obstacle should place out of the buffer zone. Furthermore, technically speaking Wukro mostly affected by manmade noise problem.

\section{ADWA Receiver Station}

Figure 7 shows land use of Adwa Receiver Station.

The Figure 7 above shows the total number and types of clutter and Knife around the buffer zone of ADWA receiver station which were affected signal strength at the receiver station. However, tree area much more encircled the receiver antenna.

Figure 8 shows effect of Knife-edge and Clutter number at Adwa receiver station.

The Figure 8 above shows the signal loss over the land use and land cover pattern of the Adwa receiver station has been recorded 3\% loss with residential, $5 \%$ loss with open land, $10 \%$ loss with tree and $1 \%$ loss with grazing land. However, the figure showed that the receiver antenna mostly affected by open land area around the antenna buffer zone. In order to have good signal strength in the receiver station, the entire obstacle should place out of the buffer zone. Further more technically speaking Adwa mostly affected by atmospheric problem.

\section{AFAR Receiver Station}

Figure 9 shows land use of Afar Receiver Station.

The Figure 9 above shows the total number and types of clutter and Knife around the buffer zone of AFAR receiver station which were affected signal 
strength at the receiver station. However, open land area much more encircled the receiver antenna.

Figure 10 shows effect of Knife-edge and Clutter number at Afar receiver station.

The Figure 10 above shows the signal loss over the land use and land cover pattern of the Adwa receiver station has been recorded 5\% loss with residential, $25 \%$ loss with open land, $3 \%$ loss with road and $1 \%$ loss with tree. However, the receiver antenna is mostly affected by open land around the antenna buffer zone. In order to have good signal strength in the receiver station, the entire obstacle should place out of the buffer zone. Further, more technically speaking afar mostly affected by Galactic noise.

\section{Result: Analysis of Clutter Loss Effect and Knife Edge Effect Analysis of Clutter Loss Effect}

"Mess misfortunes and natural commotion attributes related with different LULC classes". In arranging cell remote framework it is vital to appraise, with a high level of certainty, the mean Signal Strength after abundance tumbling off that would be gotten by singular radio beneficiary situated in different territories encompassing the site of a collector base station. Inside the developed territories this issue is muddled by shadowing impacts of tall structures and other common and man-made blocks and by the supposed blade edge number misfortune and inside a circle the Signal Strength misfortune lessened by- $6 \mathrm{~d}$ Bm and furthermore diminish as the messiness bounces from one hover to other [10]. Clutter like trees, rain, local location, and building. Agrarian land, open and sandy land (reliant on climate conditions) may likewise influence the radio wave spread specifically season. Urban communities influence the engendering of radio waves to some degree.

Trees have both retaining impact (caused by dispersing from foliage) and diffraction impact (caused by a parallel wave made by the highest point of the genuine layer), essentially for engendering over the trees. On the expression of $\mathrm{Ru}$ binstein, "mess misfortunes and natural clamor attributes related with different LULC classes" local locations have a more critical impact on radio wave engendering than do backwoods, open sandy land, farming area, meadow.

\section{The Knife-Edge Number Effect: Around All Stations}

With a specific end goal to segregate the impact of blade edge blockages and the quantity of such blockages along a way on Signal Strength quality forecast quality, alternate factors, for example, mess misfortune and separation ought to be controlled. This blockage table is extricated from the DEM document by running an Arc delineate which processes the quantity of blockages along the proliferation way to each example point [11].

From the study verified that the clutter and knife-edge that has been influenced by the radio wave engendering in the recipient station and furthermore the gathering of land utilize is very moved in Wukro station, Adwa, Quiha and Afar individually. All outcomes are given in the Geos spatial statically analysis above and it is seen that Quiha has a related environmental commotion issue; 
WUKRO related with man-made noise, Adwa is influenced by air issues while afar station has galactic noise.

\section{Resolution: ICEPAC Model Calibration Algorithm}

Build up a model that incorporates geospatial wonders in radio wave spread so as to solve radio Signal Strength loss in all station.

The Figure 11 for amendment algorithm for ICEPAC software is listed below and shown in the appendices.

The Figure 11 shows the flow chart that helps to calibrate the ICEPAC Model by introducing Geospatial Technology into the software like: GIS, Remote Sensing and GPS Data. Previously ICEPAC model only consider free space propagation elements. However from the result of research showed most Radio signal not reached receiver station because of Geospatial effect so now this flow chart overcome those mention problem by introducing Geospatial technology in order to overcome Geospatial effect.

\section{Model Calibration Algorithm: Ideal Explanation}

The new model was introduced three important factors which were lacks in the previous model. However, these three factors pointed out from the study, the first one apply site selection or suitable best receiver site selection techniques, secondly, applying Geo spatial technology, lastly applying Geo Spatial elements.

\section{Apply Site Selection Techniques Using GIS Tools}

The primary stage is utilized to figure out which station in the examination range outline course from the transmitter to the collector utilizing the introduced line calculation. Once the course was resolved, geo-spatial information was added utilizing the opposite separation weighted averaging calculation. On the off chance that the choice was chosen beforehand figured geo-spatial (separate, rise, temperature, rain fall, mugginess, daylight, twist) along the transmission course were then contrasted with the geo-spatial along the introduced line or interjected line to locate the greatest number of interference, if show.

A new model under the "receiver location finder" toolbox has been created to manage the continuous process of spatial analysis It is possible to easily experiment with parameter values, use different input data, run the model over and over again, and share it with others, if we are dealing with models to process the spatial analysis operation. The name of the new model has been assigned as "find receiver location".

The model properties dialog box. In addition to store relative path has been checked so that all paths for sources of information referenced by the tool are saved relative to the location of the toolbox specifying the Environmental settings of the newly created model. It is important to specify the environmental settings so that values specified in the environmental settings would be applied to appropriate results from running to.

\section{Applying Geo Spatial Technology}

On the off chance that the collector region is clear, support zone clearances are assessed. In the event that the messiness twofold blockage sort choice is chosen Using Remote sensing principle, at that point a hunt by utilizing controls 


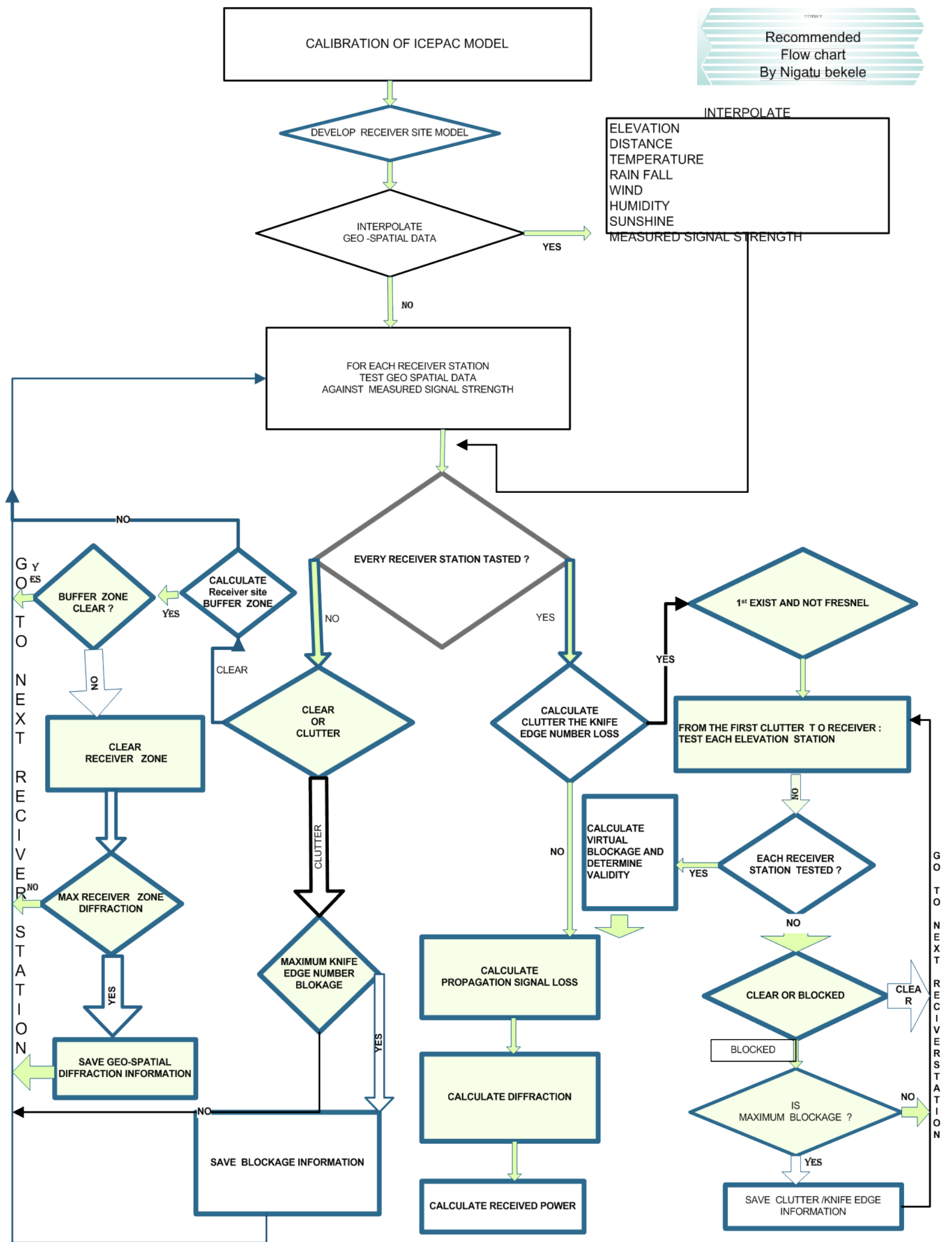

Figure 11. Amendment algorithm for ICEPAC software. 
articulation by using GPS data collection techniques. The second blockage is performed looking at geo-spatial in the inserted line or interjected line to the ascertained geo-spatial from another transmission way.

The line associating the essential impediment to the recipient, characterizes the new transmission way. On the off chance that two impediments are discovered, at that point the technique depicted in is utilized to separate a solitary virtual blockage for the diffraction pick up estimation by using GIS tools by interpolating rules. Diffraction picks up, way transmissions misfortune lastly, got influence can be ascertained, and once the above stages are finished, added geo spatial elements.

\section{Applying Geo Spatial Elements}

The stations of the investigation delineate speak to the receiver area course from the receiver area to the collector site are resolved utilizing a changed inserted line calculation and added to the new ICEPAC display. The Geo spatial factor considered in the new mode are, for example, tree, Valley. Road, residential area, industrial area, agricultural, metrological data.

Open land, cropland, building highlight classes are expressed as model parameters and their being model parameters has been shown in the exchange box of the model. Site determination procedures consolidate a more definite model of the world's surface and would oblige for multipath reflection. Certain reflected waves can touch base at the beneficiary out of stage with the essential wave. Hence any client from the yields of this information can comprehend what parameters are utilized to choose the best reasonable territories to find best radiation site.

\section{Conclusions}

Today our universe was getting worst because of unwanted particles introduced into the universe. Most of the scholars showed the effect of environmental problem onto the agricultural problem, natural resource degradation and so on, but this researches showed that environmental problem affected propagation of radio waves by attenuated and hiding transmitted signal not to reach the receiver station, for that reason, this research verified the main cause of radio wave propagation.

The research found that Geo Spatial rudiments strongly affected radio wave propagation. The main element of geo Spatial effect is, firstly, Climate element like: rain, wind, temperature, humidity, affected radio wave propagation and secondly, Clutter, knife edge and terrain. By considering geospatial effect and using Geospatial technology into the prediction Model, it is possible to exactly predict the Strength of the signal from any transmitter at any other location, if all the elements of the propagation environment are correctly taken into account.

Applying GIS, GPS and Remote sense sing technology, it is possible to select receiver station and this technology improved the signal strength at the receiver station as well as transmitter station. The research pointed out that Geo Spatial Technology (GIS, RS and GPS) oriented signal strength prediction can significant- 
ly improve prediction quality compared to the hypothetical free space model which does not take into account any local terrain feature effects, Clutter effect and Knife edge effect in general Geo spatial effect.

In summary, GEO SPATIAL technology will continue to play a vital role in Radio wave propagation system management. Any Communication network planner, prediction software developer should apply GEO SPATIAL technology for prediction signal strength and antenna site selection and also the research would be significant for an organization that launches radio signal into the environment and receiving radio signal from the environment like commercial and military broadcast station communication system. In addition to this, research consequences are important to help communication system designers in various applications such as meteorology, broadcast station radio communications, radar station, and aircraft guidance systems which will solve their challenge of predicting and compensating the potentially serious weather-related-effects.

\section{References}

[1] Anti Reddy, A.M. and Reddy, K.M. (1996) Performance Analysis of IRS Bands for Land Use/Land Cover Classification System Using Maximum Likelihood Classifier. International Journal of Remote Sensing, 17, 2505-2515. https://doi.org/10.1080/01431169608949089

[2] Jordan, M. and Chong, A. (2002) UK Planning Model for Digital Terrestrial Radio vision Coverage. IBC 99, Conference Publication, 393-398.

[3] Rappaport, T.S. (1996) Wireless Communications, Principles and Practices. Prentice Hall, PTR Upper Saddle River, New Jersey, 260.

[4] Naveenchandra, B.K.N., Lokesh, U. and Gangadhara, B.H. (2009) Importance of Geology and Soil Survey for Mobile Communication Site Planning Using RS/GIS. International Journal of Earth Sciences and Engineering, 3, 16-26.

[5] Neskovic, A., Neskovic, N. and Paunovic, G. (2000) Modern Approaches in Modeling of Radio Systems Propagation Environment. IEEE Communications Surveys \& Tutorials, 3, 2-12.

[6] Lee, W. (1982) Radio Communications Engineering. McGraw-Hill Inc., New York.

[7] Stewart, F.G. (2008) Ionospheric Communications Enhanced Profile Analysis \& Circuit (ICEPAC) Prediction Program, Technical Manual. http://elbert.its.bldrdoc.gov/pc-hf/hfwin32.html

[8] Lane, G. (2005) Review of High Frequency Ionospheric Communications Enhanced Profile Analysis \& Circuit (ICEPAC) Prediction Program. Ionospheric Effects Symposium, Alexandria, 261-1-261-8.

[9] Seybold, J.S. (2005) Introduction to RF Propagation. John Wiley and Sons, Hoboken.

[10] Rubinstein, T. (1998) Clutter Losses and Environmental Noise Characteristics Associated with Various LULC Categories. IEEE Transactions on Broadcasting, 44, 286-293.

[11] Bullington, K. (1947) Radio Propagation at Frequencies above 30 Megacycles. Proceedings of the Institute of Radio Engineers, 35, 1122-1136. https://doi.org/10.1109/JRPROC.1947.232600 